\title{
The role of bcsE gene in the pathogenicity of Salmonella
}

\author{
Caner Özdemir ${ }^{1, \dagger}$, Nefise Akçelik ${ }^{2, \ddagger}$, Fatma Neslihan Özdemir ${ }^{3, \S}$, \\ İem Evcili ${ }^{4, \uparrow}$, Tamer Kahraman ${ }^{4, \#}$, İhsan Gürsel ${ }^{4, \$}$ and Mustafa Akçelik ${ }^{5, *, !}$
}

${ }^{1}$ UNAM - National Nanotechnology Research Center, Bilkent University, 06800, Ankara, Turkey, ${ }^{2}$ Biotechnology Institute, Ankara University, Ankara, Turkey, ${ }^{3}$ Department of Food Engineering, Middle East Technical University, Ankara, Turkey, ${ }^{4}$ Department of Molecular Biology and Genetics, Bilkent University, Ankara, Turkey and ${ }^{5}$ Department of Biology, Ankara University, 06100, Ankara, Turkey

*Corresponding author: Department of Biology, Ankara University, 06100, Ankara, Turkey. Tel: +903122126040/1056; E-mail: akcelik@science.ankara.edu.tr

One sentence summary: This article describes the role of BcsE, which is a c-di-GMP binding protein, in Salmonella biofilm formation properties and pathogenicity.

Editor: Brooke Napier

${ }^{\dagger}$ Caner Özdemir, http://orcid.org/0000-0003-1259-1824

${ }^{\ddagger}$ Nefise Akçelik, http://orcid.org/0000-0001-5541-1681

§Fatma Neslihan Özdemir, http://orcid.org/0000-0003-4910-5156

‘irem Evcili, http://orcid.org/0000-0001-5455-0241

"Tamer Kahraman, http://orcid.org/0000-0002-5527-7336

\$ìhsan Gürsel, http://orcid.org/0000-0003-3761-1166

!Mustafa Akçelik, http://orcid.org/0000-0002-1227-2324

\begin{abstract}
The effects of the bcsE gene and BcsE protein on bacterial physiology and pathogenicity in Salmonella Typhimurium and Salmonella Group C1 were investigated. It was observed that biofilm and pellicle formation did not occur in the bcsE gene mutants of wild-type strains. Besides, the 'rdar' (red, dry, rough) biofilm morphotype in wild-type strains changed significantly in the mutants. In terms of the bcsE gene, the swimming and swarming motility in mutant strains showed a dramatic increase compared to the wild-type strains. The Salmonella bcsE gene was cloned into Escherichia coli BL21, and the his-tagged protein produced in this strain was purified to obtain polyclonal antibodies in BALB/c mice. The antibodies were showed labeled antigen specificity to the BscE protein. As a result of immunization and systemic persistence tests carried out with BALB/c mice, BscE protein was determined to trigger high levels of humoral and cellular responses (Th1 cytokine production, IgG2a/IgG1 > 1). Systemic persistence in the liver and spleen samples decreased by $99.99 \%$ and $100 \%$ in the bcsE mutant strains. Finally, invasion abilities on HT-29 epithelial cells of wild-type strains were utterly disappeared in their bcsE gene mutant strains.
\end{abstract}

Keywords: Salmonella; bcsE; biofilm; invasion; persistence; pathogenicity

\section{INTRODUCTION}

Salmonella is a rod-shaped, Gram-negative, facultative anaerobic bacteria belonging to the Enterobacteriaceae family (Barlow and Hall 2002). These bacteria can adapt to many animal hosts, including humans (Allerberger et al. 2003). Salmonella is identified in many foods, primarily in poultry, eggs and dairy products (Silva et al. 2011). Among other food sources that play a role in Salmonella transmission, there are also fresh fruits and vegetables (Pui et al. 2011). However, pigs, poultry and cattle are primary sources of Salmonella infection (Gillespie et al. 2005). Non-typhoidal Salmonella serovars constitute one of 
the most common pathogenic bacteria groups, with approximately 100 million foodborne cases a year worldwide (Majowicz et al. 2010). Typically, Salmonella causes self-limiting (local) gastroenteritis. However, in young, aged and immunocompromized patients, a common systemic infection can lead to septicemia and death. Some strains of Salmonella, called invasive non-typhoidal Salmonella (iNTS), have been observed to cause systemic salmonellosis (Lamps 2007).

Biofilms are communities of microorganisms that attach to a surface and behave like a multicellular organism (Mah and O'Toole 2001). Extracellular polymeric matrix (EPS) structure is characteristic in many biofilm structures. Generally, polysaccharides, proteins, nucleic acids and lipids are located in EPS structure, and they form the three-dimensional polymer network structure that provides the mechanical stability of the biofilm, its adhesive form, its attachment to a surface and the communication between the cells (Flemming and Wingender 2010). Salmonella is the most typical environmental adaptation mechanism, and persistent infection can form biofilms on biotic and abiotic surfaces.

Cellulose plays a crucial role in modulating virulence in many pathogens and being one of the main structural components of the biofilm matrix in the pathogenicity of Salmonella (Römling, Galperin and Gomelsky 2013). A total of two different operons have been identified in Salmonella, involved in cellulose biosynthesis (bcsABZC and bcsEFG). In studies conducted on the bcsA gene, which is the catalytic subunit of the cellulose synthase enzyme in these operons, it has been determined that pellicle formation decreases on solid surfaces in biofilms and liquid environments (Zogaj et al. 2001). The literature analysis showed that the BcsE protein containing the c-di-GMP binding domain takes part in a global regulation network in Salmonella (Fang et al. 2014b).

As a result of studies carried on the role of BcsE, it was suggested that the inactivation of BcsE causes a significant decrease in cellulose biosynthesis in both Escherichia coli and Salmonella Typhimurium (Solano et al. 2002; Serra, Richter and Hengge 2013). On the other hand, Fang et al. (2014a) showed that BcsE protein is not essential for cellulose biosynthesis. However, it plays a role in realizing the density and regulation of secreted cellulose fibrils (Fang et al. 2014b). The same researchers have suggested that binding to c-di-GMP via the domain called GIL (DUF2813) is critical for the BcsE protein activity. Although there are limited studies on this subject, the role of the bscE gene in biofilm and Salmonella pathogenicity has not been clarified yet.

The present study aims to determine the role of bcsE in Salmonella pathogenicity. We aimed to investigate the bcsE gene involved in Salmonella pathogenesis better and advanced with new agents with substantial potential in combating Salmonella infections.

\section{MATERIAL AND METHODS}

\section{Bacterial strains and culture conditions}

Biofilm producer strain, which was isolated poultry meat from Turkey, Salmonella enterica Group C1 (Serogroup C1, strain code DMC2), Salmonella enterica Typhimurium (strain code DMC4) strains, c $\triangle \mathrm{DMC2}$ and $\mathrm{c} \triangle \mathrm{DMC} 4(\triangle \mathrm{bcsE}$ pBAD::bcsE) complemented strains, E. coli BL21, Staphylococcus aureus, Enterococcus faecalis strains used in the study, were obtained from the Prokaryotic Genetics Laboratory of the Biology Department Faculty of Science, Ankara University. Strains were mixed with $60 \%$ (v/v) glycerol supplemented Luria Bertani (LB) broth (Merck, Germany; final concentration $30 \% \mathrm{v} / \mathrm{v}$ ) and stored at $-80^{\circ} \mathrm{C}$. Bacteria were prepared for all the experiments by inoculation in LB broth and incubated at $37^{\circ} \mathrm{C}$ for $18 \mathrm{~h} 200 \mathrm{rpm} / \mathrm{min}$ under shaking conditions. For biofilm experiments, $\mathrm{LB}$ broth without $\mathrm{NaCl}\left(\mathrm{LB}^{-\mathrm{NaCl}}\right)$ was used as test broth.

\section{Construction of bscE gene deletion mutant}

Deletion of the bcsE gene from the chromosome of $\mathrm{S}$. Typhimurium (DMC4) and S. Group C1 (DMC2) was performed using the standard lambda red recombinase method as previously described (Datsenko and Wanner 2000). Briefly, at the first step, the chloramphenicol (Chl) resistance cassette was amplified from template plasmid pKD3, with homology arms to the bcsE gene. Red recombinase expressed pKD46 plasmid was transformed into the test Salmonella strains to perform chromosomal gene mutations via homolog recombination. Then, the amplified antibiotic cassette was transformed into Salmonella strains by electroporation. Red/ET expressing genes were induced by $50 \mu \mathrm{L} 10 \% \mathrm{~L}$-arabinose into the growth media. After the incubation at $37^{\circ} \mathrm{C}$ for $1 \mathrm{~h}, 100 \mu \mathrm{L}$ of solution were inoculated on LB agar medium containing chloramphenicol $(25 \mu \mathrm{g} / \mathrm{mL})$. Colonies were selected for PCR confirmation after overnight incubation at $37^{\circ} \mathrm{C}$. For this purpose, the primer sequences (F 5'-tcggcatctcatcattatgg-3' and R 5'caatcgcaggggttcaggaa-3') used in the PCR reaction have been designed based on each homologous arms used in recombination.

\section{Phenotypic tests}

Biofilm morphotypes of S. Typhimurium (DMC4), S. Group C1 (DMC2) strains were determined on LB agar media (without $\mathrm{NaCl}$ ) containing Congo red (Römling and Rohde 1999). The presence of cellulose at biofilm structure to determined $10 \mu \mathrm{L}$ of the test strains $\left(\mathrm{OD}_{595}=0.2\right)$ were inoculated on the calcofluor (fluorescent brightener 28, Sigma-Aldrich, China, $200 \mu \mathrm{g} / \mathrm{L}$ ) supplemented $\mathrm{LB}^{-\mathrm{NaCl}}$ agar medium. Plates were incubated for 8 days at $20^{\circ} \mathrm{C}$. The presence of cellulose in the biofilm structures of the strains was evaluated under $366 \mathrm{~nm}$ UV light. Images were obtained by using the Gel Logic 200 Imaging System (Kodak, Oregon).

The methods to analyze the swimming and swarming motilities of bacteria, recommended by Ahmad et al. (2016) were used. Swimming motility was analyzed LB agar plate. A single colony was removed from the overnight culture on the agar surface and inoculated on $0.3 \% \mathrm{LB}$ agar surface and incubated for $5 \mathrm{~h}$ at $28^{\circ} \mathrm{C}$ and $4 \mathrm{~h}$ at $37^{\circ} \mathrm{C}$, respectively. Swarming motility was analyzed similarly incubated overnight on LB agar medium at $37^{\circ} \mathrm{C}$. At the end of the incubation, a single colony was taken from the agar surface and inoculated into a $0.5 \% \mathrm{LB}$ agar medium containing $0.5 \%$ glucose. Agar plates were incubated for $5 \mathrm{~h}$ at $28^{\circ} \mathrm{C}$ and 4 $\mathrm{h}$ at $37^{\circ} \mathrm{C}$, respectively. The diameters of halos due to bacterial migration were measured $4 \mathrm{~h}$ post-inoculation.

The pellicle forming properties examined at the $S$. Typhimurium (DMC4) and S. Group C1 (DMC2) strains, $0.5 \mathrm{~mL}$ portions taken from overnight cultures were inoculated into $4.5 \mathrm{~mL} \mathrm{LB}-\mathrm{NaCl}$ broth and incubated for 8 days at 20 and $37^{\circ} \mathrm{C}$. Pellicle formation of the strains was observed daily (Solano et al. 2002) to determine ring formation in the liquid-air intermediate phase and cell accumulation at the bottom of the glass tubes (Römling et al. 2000). 


\section{Biofilm production on polystyrene surfaces}

Biofilm production ability of Salmonella strains was tested using 96-well polystyrene microdilution plates. Test strains were grown in $5 \mathrm{~mL} \mathrm{LB}{ }^{-\mathrm{NaCl}}$ broth at $37^{\circ} \mathrm{C}$ and agitation $(200 \mathrm{rpm} / \mathrm{min})$ for $18 \mathrm{~h}$. A total of $30 \mu \mathrm{L}$ of active cultures were transferred into wells containing $100 \mu \mathrm{L}$ of $\mathrm{LB}^{-\mathrm{NaCl}}$ broth to was determined the optimal biofilm production. Microtiter plates containing bacterial suspensions were incubated for 24, 48, 72, 96, 120 and 144 $\mathrm{h}$ at $20^{\circ} \mathrm{C}$ under static conditions. At the end of the incubation period, unattached cells were removed by washing wells three times with sterile physiological serum $(0.85 \% \mathrm{NaCl})$ (Merck, Germany). Plates were dried at room temperature, and the biofilm matrix was fixed by $95 \%$ methanol addition for $15 \mathrm{~min}$. The fixed structure was stained with $130 \mu \mathrm{L}$ of $0.1 \%$ crystal violet dye and incubated at room temperature for $30 \mathrm{~min}$. Wells were washed two times with sterile distilled water to remove the unbounded dye. The dyes bound was dissolved in the biofilm matrix, 130 $\mu \mathrm{L}$ of $33 \%$ glacial acetic acid was transferred to the wells and the plates were incubated for $45 \mathrm{~min}$ at room temperature. The optical density of the dissolved dye was measured by Elisa reader (Biorad, California, USA) at $\mathrm{OD}_{595} \mathrm{~nm}$. The experiment was carried out with four parallel and two replicates for all strains. The biofilm measurements were calculated by averaging the OD values of the control wells (wells containing only LB liquid medium without $\mathrm{NaCl}$ ) from the average of the OD values from parallels. Strains were also evaluated as 'non producers', 'weak producers', 'intermediate producers' and 'strong producers' in terms of biofilm production based on the conversions of 'cut off' (limit) values (Stepanović et al. 2000).

\section{Cloning and heterologous production of BcsE protein}

The pBAD24 vector was used for complementation studies by cloning bcsE downstream of the arabinose inducible BAD promoter. Primer pairs to be used in the amplification of the bcsE gene from S. Typhimurium DMC2 and DMC4 were designed by adding PstI and SalI restriction endonuclease recognition sequences (bcsE-N-ter-forward primer: 5'-ctcctgcagtcggcatctcatcattatgg-3' PstI and bcsE-N-ter-reverse primer 5'- gaggtcgaccaatcgcaggggttcaggaa -3' SalI). Amplified bcsE gene was cloned to pBAD24 after PstI end SalI cleavage, and the recombinant vector was transformed into bcsE gene mutant strain of S. Typhimurium DMC2 and DMC4.

An expression vector, pET28a, was used to produce the BcsE protein in a heterologous host. Restriction endonuclease recognition sequences were added to primer sequences designed to amplify the bcsE gene-specific region (bcsE-N-terforward primer:5'- ctcgaattctcggcatctcatcattatgg-3'; bcsE-N-terreverse primer 5'- gaggtcgaccaatcgcaggggttcaggaa -3') and PCR amplifications were performed. SalI and EcoRI are making a single cut which opens the circular form in the pET28a vector, and the linear molecule, formed, has sticky ends.

The recombination of the enzyme cleaved PCR product with the linearized PET28a was performed by a ligation reaction. In the ligation reaction, the vector/insert ratio was set to be $3 / 1$. The recombinant vector obtained by cloning the bcsE gene into the pET28a vector was transformed into the E. coli BL21 strain. Recombinant BL21 (BL21 ${ }^{\mathrm{Rc}}$ ) strain was induced with isopropyl$\beta$-D-thiogalactopyranosoid (IPTG). The culture was incubated in a B-PER bacterial cell lysis reagent (Thermo Scientific, Rockford, USA) for $10 \mathrm{~min}$ at room temperature on the rotator. After this step, poly-histidine labeled proteins were purified by following the HisPur Ni-NTA purification kit (Thermo Scientific) method steps. The sodium dodecyl sulfate-polyacrylamide gel electrophoresis (SDS-PAGE) method was used to observe his-taglabeled BcsE protein expression recombinant strain.

The PierceTM BCA Protein Assay Kit (Thermo Scientific) was used to determine the concentration of purified BcsE protein to immunize BALB/c mice. Bovine serum albumin (BSA) was used as a standard. BSA concentrations in the range of $0-2000 \mu \mathrm{g} / \mathrm{mL}$ and standard working reagent were prepared according to the kit protocol. A total of $25 \mu \mathrm{L}$ of the standard solution and the protein samples to be measured were added in parallel to the 96-well microtiter plates, and then $200 \mu \mathrm{L}$ of reagent was transferred. After shaking the microtiter plate for $30 \mathrm{~s}$, it was left to incubate for $30 \mathrm{~min}$ at $37^{\circ} \mathrm{C}$. After the incubation, the absorbance of the samples was measured in the ELISA reader at $562 \mathrm{~nm}$ wavelength. With the standard curve obtained from BSA standards of known concentration, the protein concentrations used to BALB/C mice in the immunization study were determined.

\section{Polyclonal antibody production}

A total of two male BALB/c mice, 6-8-weeks-old, were used to produce polyclonal antibodies. Recombinant BcsE protein $(50 \mu \mathrm{g} / \mathrm{mL})$ and immunostimulant ODN1466 and ODN1555 adjuvants $(40 \mu \mathrm{g} / \mathrm{mL})$ were emulsified to increase the immune response in mice. This emulsion was injected intraperitoneally in mice on days 14 and 28. Blood was collected from the tail vein of the mice before injection, and sera was stored at $-20^{\circ} \mathrm{C}$ for further analysis. Similarly, sera samples were collected at the 2nd and 4th weeks after injection (Xie et al. 2005). Blood samples were incubated for $1 \mathrm{~h}$ at $37^{\circ} \mathrm{C}$ and centrifuged at $2000 \mathrm{~g}$ for 5 min. Total IgG, IgG1 and IgG2a immunization from sera samples obtained were evaluated by the ELISA method.

To test the polyclonal antibody-antigen specificity, purified BcsE protein samples, DMC2, DMC4, $\triangle \mathrm{DMC} 2, \triangle \mathrm{DMC} 4$, E. coli BL21, S. aureus, E. faecalis strains were tested against the polyclonal antibody using the western blot technique (Laemmli 1970). The Institutional Animal Care and Use Committee of the Department of Molecular Biology and Genetics (Bilkent University, Ankara, Turkey) have approved all procedures (Approval \#: BILAEC NO:2015/38). All experimental protocols used in this study were performed according to the 'Guide for the Care and Use of Laboratory Animals' published by the National Institutes of Health and were conducted according to the international regulations and declarations concerning animal experimentation.

\section{Anti BcsE IgG ELISA}

BcsE protein samples were transferred to high binding polystyrene microtiter plate wells in $50 \mu \mathrm{L}$ PBS buffer with a final concentration of $5 \mu \mathrm{g} / \mathrm{mL}$ and incubated at room temperature for $4 \mathrm{~h}$. After the incubation, the wells were washed three times with $0.1 \%$ PBS-T and blocked with the addition of Blocking Buffer (1X PBS with 5\% BSA and 0,05\% Tween-20). A total of $50 \mu \mathrm{L}$ of 4 -fold serially diluted sera samples, starting from $7 \mathrm{X}$ to $114688 \mathrm{X}$ titration, were transferred to microtiter plates and incubated at room temperature for $4 \mathrm{~h}$. Following the incubation, plates were washed three times with $0.1 \%$ PBS-T. Afterward, $50 \mu \mathrm{L}$ of $1 \mu \mathrm{g} / \mathrm{mL}$ alkaline phosphatase-conjugated total IgG, IgG1 and IgG2a antibodies were added to the plates and incubated for $1 \mathrm{~h}$ at room temperature. After incubation, the plates were washed as described above, and PNPP substrate solution was added to the wells. The color development was observed, and the optical density of samples was measured by ELISA reader at $\mathrm{OD}_{405} \mathrm{~nm}$ wavelength. 


\section{BALB/c infection model}

A competition experiment was performed in 6-8-weeksold male BALB/c mice using wild-type (DMC2-DMC4) and mutant ( $\triangle \mathrm{DMC} 2-\triangle \mathrm{DMC}$ ) Salmonella strains. The cell density of overnight Salmonella cultures was adjusted to $10^{6} \mathrm{cells} / \mu \mathrm{L}$ in $\mathrm{PBS}$, and $100 \mu \mathrm{L}$ of this solution was overnight bacterial cultures mixed with PBS in a ratio of $1: 1$ and $10^{6}$ cells $/ 100 \mu \mathrm{L}$ were administered intraperitoneally to mice. On the 1st and 3rd days of the application, the spleen and livers were collected and homogenized in PBS. The competitive index (CI) was calculated by planting the obtained suspensions on the agar plate surface (Beuzón and Holden 2001). In the experiments, the groups were as follows:

- Control group ( $n=5): 100 \mu \mathrm{L}$ PBS

- Infected group $(n=5): 1 \times 10^{6}$ cells per $100 \mu \mathrm{L}$ of wild-type strain was prepared DMC2 and $\triangle \mathrm{DMC} 2$

- Infected group $(n=5): 1 \times 10^{6}$ cells per $100 \mu \mathrm{L}$ of wild-type strain was prepared as DMC4 and $\triangle \mathrm{DMC} 4$ (Beuzón and Holden 2001).

\section{Human epithelial cells invasion experiment}

The human epithelial cell line HT-29 (colon, colorectal adenocarcinoma) was incubated in 24 -well plates at $37^{\circ} \mathrm{C}$ by adding $2 \mathrm{mM}$ L-Glutamine, 1\% Penicillin/Streptomycin, 10\% FBS to the RPMI medium.

A total of $10^{6} \mathrm{CFU} / \mathrm{mL}$ of wild-type and mutant cells were inoculated on the epithelial cell $\left(10^{5}\right.$ cells/well) line and incubated for $1 \mathrm{~h}$. Following the incubation, a medium containing $100 \mu \mathrm{g} / \mathrm{mL}$ gentamicin was transferred to cells to kill extracellular bacteria. After the cells were washed twice with PBS containing 1\% Triton X-100, the number of intracellular bacteria was determined as the colony-forming unit on agar plates (SteeleMortimer 2007).

In addition to this method, wild-type and mutant strains are labeled with a lipophilic dye, SP-DiOC $(5 \mu \mathrm{g} / \mathrm{mL})$, which is excited by the blue laser and emits light at $515 \mathrm{~nm}$. Viability was determined with propidium iodide $(2 \mu \mathrm{g} / \mathrm{mL})$ in the flow cytometer, and $2 \times 10^{6} \mathrm{CFU} / \mathrm{mL}$ was placed on the bacterial cell/epithelial cell ( $10^{5}$ cells/well) line and incubated for $1 \mathrm{~h}$. The wells were washed three times with PBS, and a medium containing $100 \mu \mathrm{g} / \mathrm{mL}$ gentamicin (>64 $\mu \mathrm{g} / \mathrm{mL}, \mathrm{MIC})$. was added to remove and kill extracellular bacteria.

\section{Statistical analysis}

Statistical analyzes were carried out using GraphPad Prism 8 software. Biofilm production capacity, competition experiments, IgG2a/IgG1 ratio and human epithelial cell invasion were determined using the ' $F$ ' value of the results obtained from the experiments; a one-way ANOVA test was applied to determine whether the differences between the groups were statistically significant. Tukey accuracy test was used in the evaluation of variations between groups.

\section{RESULTS}

\section{Phenotypic evaluation of bcsE mutants}

Colony PCR was performed using primers that replicated the chloramphenicol gene cassette with mutant strains that developed in agar medium containing chloramphenicol. The mutation was confirmed by amplifying the gene cassette.
As a result of tests performed on LB agar containing Congo red, biofilms formed by wild-type S. Typhimurium (DMC4), S. Group C1 (DMC2) strains were determined to be in the 'rdar' (red, dry and rough) morphotype. This morphotype development was also observed as 'rdar', including bcsE gene complemented mutants. It was determined that the biofilm structures formed by Salmonella strains mutant in terms of bcsE gene tend to disintegrate. This structure is similar to the 'bdar' (brown, dry and rough) morphotype (Fig. 1A).

The production of cellulose in Salmonella strains was investigated qualitatively in LB agar containing calcofluor, and it was determined that cellulose production in mutant strains decreased compared to wild-type strains and including bcsE gene complemented mutants under $366 \mathrm{~nm}$ UV light (Fig. 1B).

As an exciting result in the bcsE gene mutants of both Salmonella strains investigated, it was determined that both swimming and swarming motility showed a significant increase compared to wild-type strains and bcsE gene complemented mutants (Fig. 1C and D).

As a result of the trials of comparing the pellicle structures, it was determined that wild-type and bcsE gene complemented mutants produced a highly rigid pellicle. In contrast mutant strains could not form pellicles following the incubation at $20^{\circ} \mathrm{C}$ (Fig. 1E).

The biofilms formed by S. Group C1 (DMC2) and S. Typhimurium (DMC4) wild-type strains, bcsE mutants and bcsE gene complemented mutant strains in microtiter plates on different days are given in Fig. 2. According to the obtained data and calculated threshold values, the maximum biofilm production was $120 \mathrm{~h}$ for both serotypes. After $120 \mathrm{~h}$, it was determined that S. Group C1 (DMC2) strain with $\mathrm{OD}_{595}=2.797$ and S. Typhimurium (DMC4) strain with $\mathrm{OD}_{595}=3.198$ values were observed to be strong biofilm producers. bcsE gene complemented mutant strains also recovered the same biofilm characteristics and strong biofilm producers as the wild-type strains $\left(\mathrm{OD}_{595}=2.777\right.$ and $\left.\mathrm{OD}_{595}=3.199\right)$, respectively. Biofilm production amount decreased as the incubation period increased. Besides, it was determined that both serotypes that are mutant in terms of bcsE did not produce biofilms in all incubation periods tested.

\section{Purification of BcsE protein}

In the purification process of $\mathrm{BcsE}$ protein from $\mathrm{BL} 21^{\mathrm{Rc}}$ strain, the immobile metal affinity chromatography (IMAC) method was applied. In this system, a strong bond is formed between the target protein labeled histidine and nickel metals in the colon. In this way, the protein to be purified clings to the column. The target protein is obtained by treatment with imidazole (Cuatrecasas 1970). Since the protein obtained in the purification process is not $100 \%$ pure, the D-salt column (Thermo Scientific) was used to purify the imidazole after the column step. The sodium dodecyl sulfate-polyacrylamide gel electrophoresis (SDS-PAGE) method was used to confirm the target protein. The western blot technique was also used to test whether the target antigen binds to the correct antibody. The gel pattern obtained shows the pure BcsE protein of $59 \mathrm{kDa}$ size (Fig. 3).

\section{Detection of immunization with anti BcsE IgG ELISA method}

In this experiment, 6-8-week-old female BALB/c mice were intraperitoneally injected with pure recombinant BcsE protein, and immunization was confirmed by antibody titration. The 


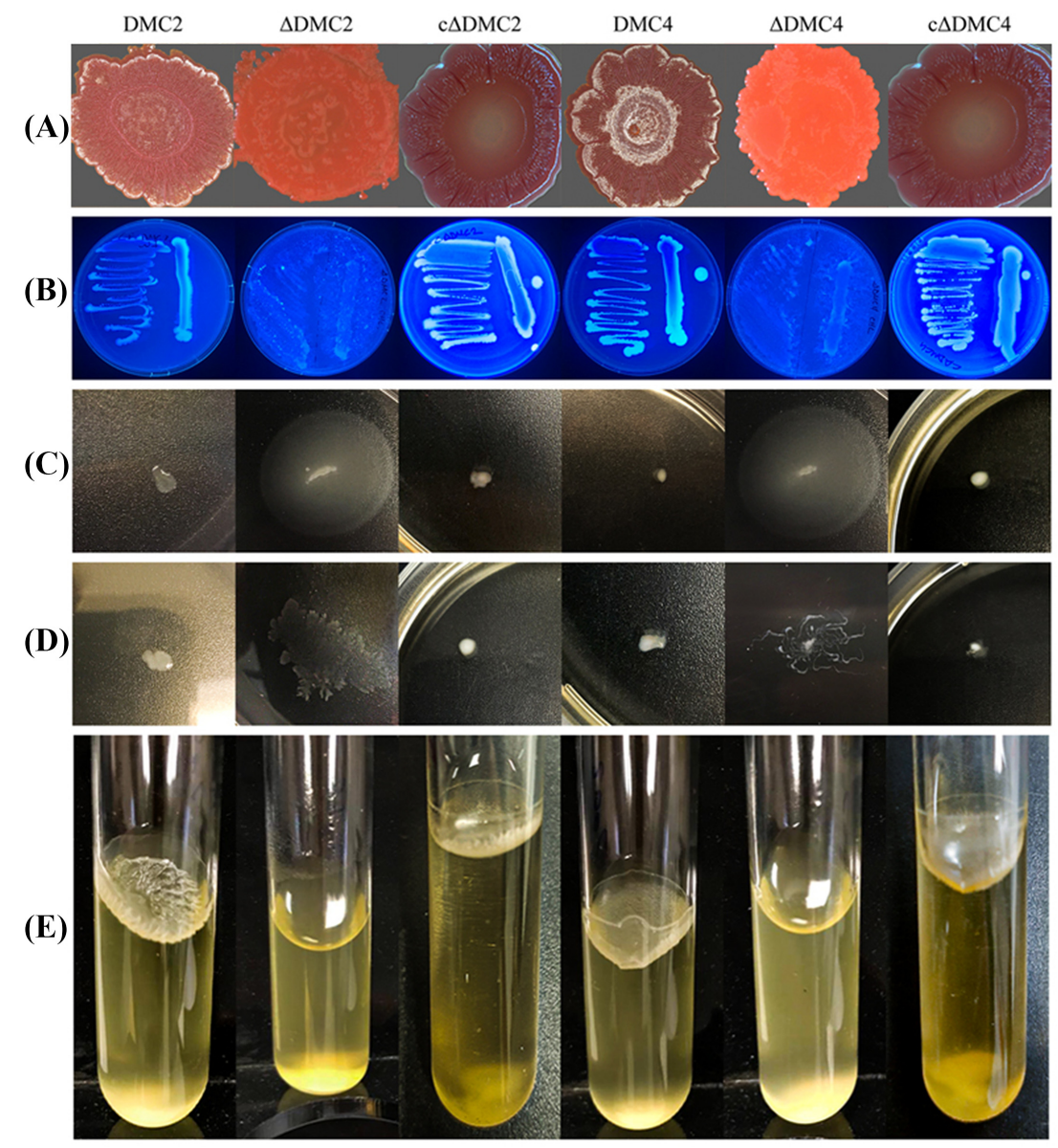

Figure 1. Biofilm phenotypes of the bcsE mutants of S. Group C1 DMC2 and S. Typhimurium DMC4 wild-type strains, the bcsE mutants and their complemented strains. (A) Stereo-microscope images of biofilm morphotypes. (B) Calcofluor binding of the bcsE mutants of Salmonella decrease compared to wild-type and their complemented strains. (C) Swimming motility $\triangle \mathrm{DMC}$ (17.50 mm), $\triangle \mathrm{DMC} 4$ (11.75 mm) (D) Swarming motility $\triangle \mathrm{DMC}$ (19.25 mm), $\triangle \mathrm{DMC} 4$ (13.00 mm) (E) Pellicle-forming properties on glass tubes.

individual response of mice for each IgG subtype at the 2 nd and 4 th post booster was analyzed. The average immune response at an indicated ratio at 1792 reciprocal dilution of mice sera dilutions for each group and IgG subtype is given in Fig. 4 ( $P$ $<0.001)$. As a result of the ELISA test, it was determined that the polyclonal antibody sera worked up to 1/114 688 dilution. IgG1 and IgG2a immunoglobulin isotypes are used to determine whether the immune response is Th1 or Th2. The IgG2a and IgG1 ratio greater than 1 indicates that the Th1 immune response is strong (IgG2a/IgG1 > 1). In our study, it was determined that the rates for each dilution were above 1 . As a result, it was determined that polyclonal antibodies from mice against BcsE protein caused immunization by generating Th1 cellular responses and IgG1 and IgG2a humoral immune responses all dilution titers (Figs 4 and 5).

Whether the antibody binds to the correct antigen was determined by western blot analysis using bacterial cell lysates and recombinant BcsE protein. The binding of antibodies to BcsE protein was achieved in wild-type DMC2 and DMC4 and E. coli BL21 strains. However, this reaction did not occur in S. aureus and E. faecalis strains used as a negative control; bcsE gene deleted DMC2 and DMC4 mutant strains. Figure 6 indicates that the polyclonal antibody shows marked antigen specificity.

\section{Competition experiments}

Competition experiments between wild-type and bcsE mutant strains were performed using 6-8-week-old male BALB/c mice. Mice were inoculated with a mixture of $\sim 2 \times 10^{6} \mathrm{cfu} / \mathrm{mL}$ each of wild-type and bcsE mutant Salmonella strains. Spleen and liver samples collected on the 1st and 3rd days after infection were homogenized and inoculated on LB agar surfaces with and without Chl, and live-cell numbers were determined due to the incubation period. The data obtained from competition experiments showed that $b c s E$ mutant strains are highly disadvantageous compared to wild-type strains in Salmonella virulence. On day 1, wild-type DMC2 and DMC4 strains were determined as $7.3 \times 10^{3}$ and $2.2 \times 10^{5} \mathrm{CFU} / \mathrm{g}$, respectively. On day 3, the wild-type DMC2 strain was $7.4 \times 10^{4} \mathrm{CFU} / \mathrm{g}$, and the DMC4 strain was $3.41 \times 10^{7} \mathrm{CFU} / g$. bcsE mutants did not colonize the agar surface (Fig. 7). Colonies counted in wild-type DMC2 and DMC4 strains on day 1 were determined as $1.47 \times 10^{6}$ and $8.1 \times 10^{5} \mathrm{CFU} / \mathrm{g}$, respectively. On day 3, the wild-type DMC2 strain was $1.30 \times 10^{5} \mathrm{CFU} / \mathrm{g}$, and the DMC4 strain was determined as $2.83 \times 10^{7} \mathrm{CFU} / g$. Regarding the bcsE gene, the mutant DMC2 and DMC4 strains were determined as $2.5 \times 10^{2} \mathrm{CFU} / \mathrm{g}$ and $4.6 \times 10^{2} \mathrm{CFU} / \mathrm{g}$ respectively on the 1 st day, $3 \mathrm{rd}$ day, $3.7 \times 10^{2} \mathrm{CFU} / \mathrm{g}$ and $4.5 \times 10^{2} \mathrm{CFU} / \mathrm{g}$ colony formation were observed, respectively 


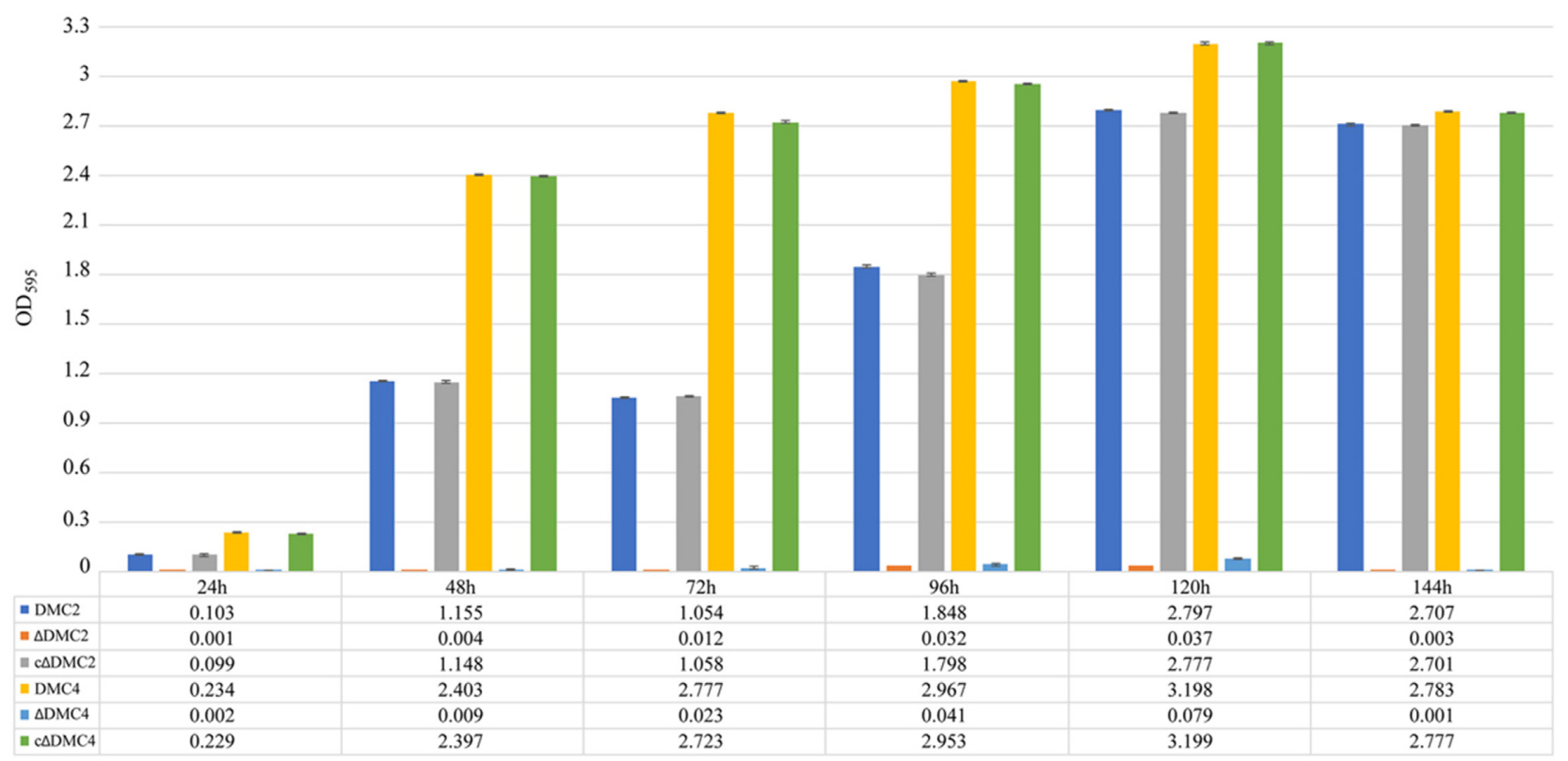

Figure 2. Biofilm production capacities of wild-type and bcsE gene deleted mutants and bcsE gene complemented mutants of Salmonella strains. One-way ANOVA compared biofilm formation on polystyrene wells from three independent experiments $(P<0.001)$ and followed by Tukey's multiple comparison test, which shows significantly different from corresponding wild-type versus bcsE gen deleted mutants.

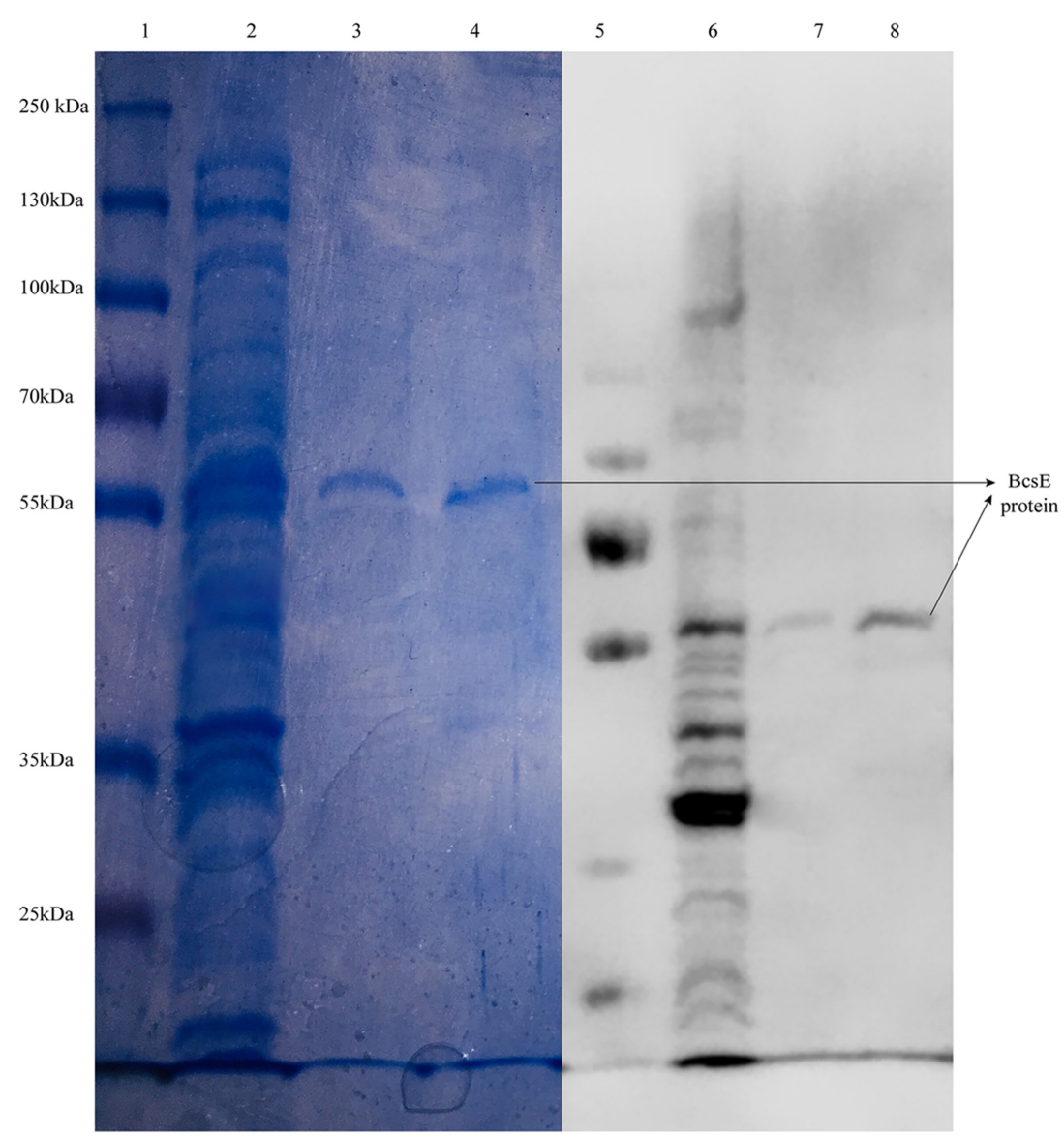

Figure 3. SDS-PAGE (wells 1, 2, 3 and 4) and western blot patterns (wells 5, 6, 7 and 8) of purified BcsE protein. (1) Protein marker, (2) Recombinant E.coli BL21 strain, (3) Equilibration buffer, (4) Elution buffer, (5) Protein marker, (6) Recombinant E.coli BL21 strain, (7) Equilibration buffer and (8) Elution buffer. 
UnRx
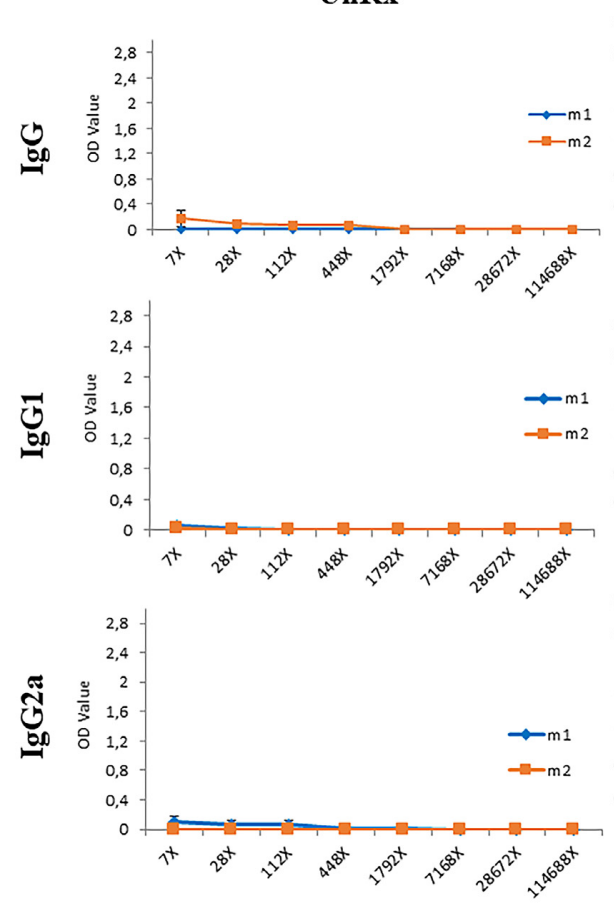

Primary
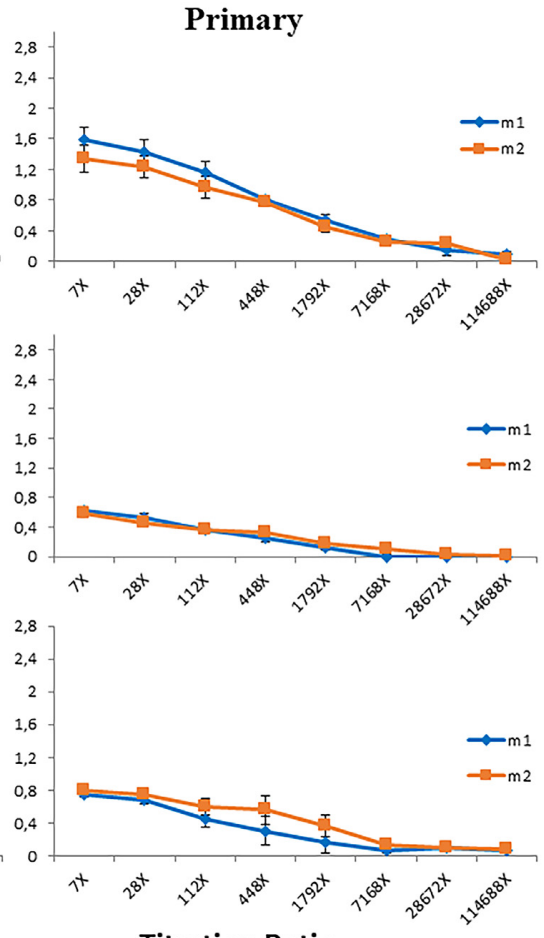

Titration Ratio

Figure 4. Sera were collected BcsE vaccinated mice at 4 weeks after post-injection (m1: mice1, m2: mice2). Average OD readings of total IgG, IgG1 and IgG2a titers indicated dilutions.

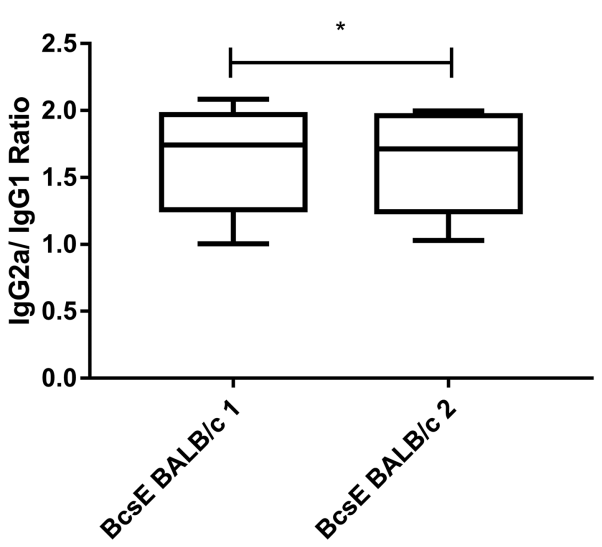

Figure 5. Box-plot of IgG2a/IgG1 ratio reciprocal dilution of indicated comparisons two mice sera.

(Fig. 8). The competitive index was calculated as the output ratio of $b c s E$ mutant to the wild-type strain, divided by the input ratio. In spleen bcsE mutant strain output is ' 0 '. Therefore, CI in the spleen could not be calculated. In liver, CI wild-type DMC2 versus bcsE mutant DMC2 1.99; wild-type DMC4 versus bcsE mutant DMC4 2 806. $\log _{10} \mathrm{CI}>0$ indicates DMC2, and DMC4 strains have a competitive advantage over bcsE mutant DMC2 and bcsE mutant DMC4 strains. One-way ANOVA compared output bacterial strains $(P<<0.05 i>)$ and followed by Tukey's multiple comparison test, which indicated significant differences between strains. For all tests, a $P<\mathrm{i}<0$. $>05$ was agreed with statistically significant.

\section{Human epithelial cell invasion}

The invasion rate of the DMC2 strain was determined as $24.6 \%$, and it was $26.2 \%$ for the DMC4 strain. bcsE gene complemented

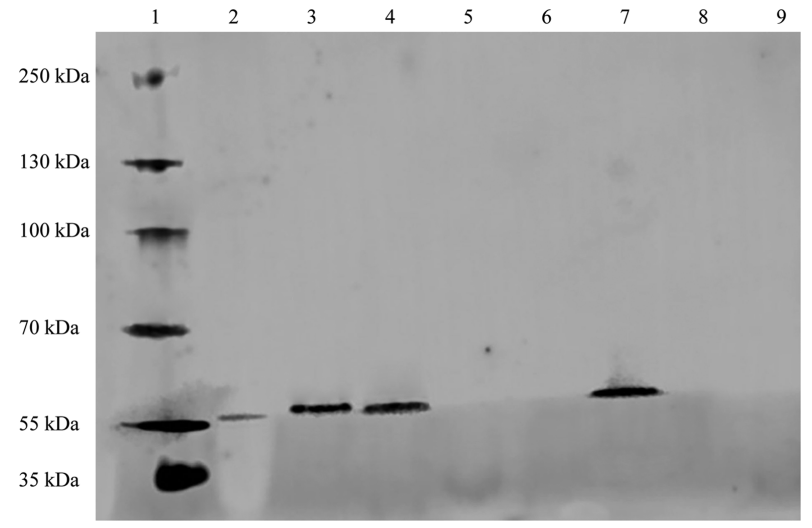

Figure 6. Western blot analysis showing polyclonal antibody specificity. (1) Protein marker, (2) BcsE recombinant protein, (3) DMC2, (4) DMC4, (5) $\triangle \mathrm{DMC} 2,(6)$ $\triangle \mathrm{DMC} 4,(7)$ E. coli BL21, (8) S. aureus and (9) E. faecalis.

DMC2 (18.98\%) and DMC4 (17.1\%) strains were also invade a eukaryotic monolayer, though at a rate that lower than wildtype strains. Invasion of bcsE mutant strains into HT-29 cells was not observed. Cell counts were performed in flow cytometry, and it was confirmed that bcsE mutant strains did not invade HT-29 cells (Fig. 9A). These results show that the bcsE gene plays an essential role in the invasion of the epithelial cell. The ability of Salmonella invasion was determined by using the HT-29 cell line. Data show mean combined results of two independent experiments with three replicates. The invasion rate of bcsE mutant differed from that wild-type and $b c s E$ gene complemented at $P<0.001$. Statistical analyzes were made with dependent one-way ANOVA using Prism 7 (GraphPad) software. 


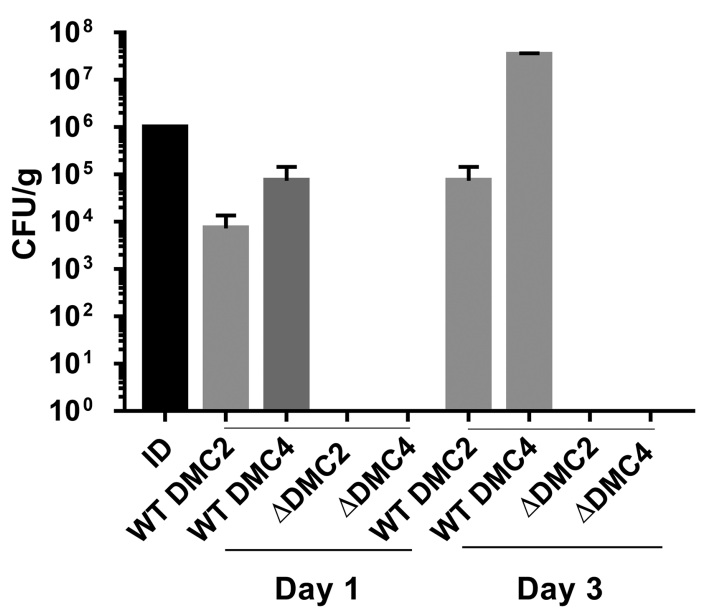

Figure 7. Viable cell counts of wild-type bcsE mutant strains on day 1 and 3 in spleen $(\mathrm{P}<<0.05 \mathrm{i}>)$.

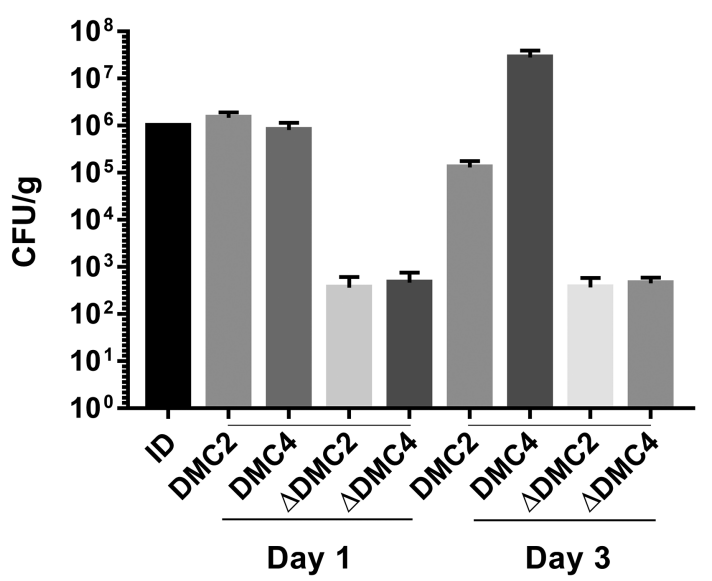

Figure 8. Viable cell counts of wild-type and their bcsE mutant strains on day 1 and 3 in liver $(P<<0.05 i>)$.

\section{DISCUSSION}

The ability of Salmonella strains to form biofilms on biotic (such as fresh fruits and vegetables, animal epithelial cells) and abiotic (such as plastic, glass and stainless steel) surfaces significantly contributes to their persistence and virulence. By forming a biofilm structure, Salmonella strains can carry out mucosal infections and develop persistent infections. The biofilm morphotype generally defined in Salmonella is 'rdar' (red, dry and rough; biofilm matrix main components are cellulose and curli fimbria). Alongside this dominant morphotype, 'pdar' (pink, dry and rough; biofilm matrix main component is cellulose), 'bdar' (brown, dry and rough; biofilm matrix main component is curli fimbria) and 'saw' (smooth and white; no curli fimbria and cellulose in biofilm matrix) morphotypes have also been described (Steenackers et al. 2012). As a result of the examinations performed in the wild-type strains used in our study, it was determined that the biofilm morphotype of wild-type strains and bcsE gene complemented mutants of S. Typhimurium (DMC4) and S. Group C1 (DMC2) was 'rdar'. However, biofilm morphotypes of the bcsE gene deleted mutants were similar to the 'bdar' biofilm morphotype. The semi-quantitative calcofluor method investigated the cellulose content of the wild-type and bcsE mutant strains biofilms. It was determined that cellulose production in mutants decreased significantly. Besides, pellicle formation properties of wild-type strains and their bcsE gene mutants in a liquid-air intermediate phase were examined. It was determined that pellicle formation (rigid and containing ring structure) was observed in wild-type and bcsE gene complemented strains. At the same time, this feature was lost in bcsE mutants. One of the essential components in the formation of pellicle structures is cellulose. In some bacteria, even pellicle structures formed by cellulose have been identified (Römling 2005). Finally, when the biofilm formation characteristics of wild-type strains and their bcsE gene deleted mutants on polystyrene surfaces were quantitatively examined, it was determined that the wildtype strains were strong biofilm producers. In contrast, mutant strains could not produce detectable biofilms. In agar environments, mutants show a biofilm phenotype like 'bdar', while their inability to produce biofilms on polystyrene surfaces can be seen as a contradiction between the two tests. However, it is typical for colonial morphology occurring in solid nutrient media to be mixed with biofilm morphotypes due to visual examinations. Therefore, the primary reference in biofilm production is the quantitative definition of biofilm formation on polystyrene surfaces. When all these data are evaluated together, it turns out that the bcsE gene encodes a mandatory regulator protein for biofilm formation in solid-air and liquid air intermediate phases in Salmonella strains studied. These data are the first to describe the direct relationship of the bcsE gene with biofilm production. The main mechanism in the appearance of this result is that the BcsE protein encoded by the bcsE gene plays an essential role in cellulose biosynthesis. Because in our findings, the main difference between wild-type strains and mutants was determined in terms of cellulose production. In Salmonella, the operons bcsABZC and bcsEFG controlled by the regulator AdrA are responsible for bacterial cellulose production (Römling 2005). In a limited number of studies conducted to date, there is evidence to support that the bcsE gene is mandatory for cellulose synthesis in S. Typhimurium and E. coli (Solano et al. 2002; Serra, Richter and Hengge 2013), as well as evidence supporting that this gene is not essential for cellulose synthesis but regulates maximum cellulose synthesis (Fang et al. 2014a). According to our study data, cellulose biosynthesis did not completely stop in the bcsE gene mutants but was significantly reduced. These data support the findings of Fang et al. (2014b).

In our study, another characteristic of S. Typhimurium (DMC4) and S. Group C1 (DMC2) wild-type strains and their bcsE gene deleted mutants were individual bacterial motility and multicellular coordinated motility differences. These differences also disappeared by reverting to the wild-type phenotype in bcsE gene complemented mutants. Bacteria inoculated in agar-containing nutrient media move to nutrient-rich areas parallel with the depletion of nutrients in the first planting region. This motility in the direction of the nutrient gradient can be independent motility of each cell in the colony via flagella (swimming) or coordinated motility of many cells (swarming). Thus, the swarming motility is also called bacterial multicellular motility. While the swimming motility is carried out through the rotation of the bacterial flagella, the swarming motility is mainly carried out through biosurfactants produced by bacterial cells (Römling 2005; Zorraquino et al. 2013; Le Guyon et al. 2015). Zorraquino et al. (2013) suggested that the increase in cellulose production was an essential factor in preventing flagellar motility (Zorraquino et al. 2013). On the other hand, swimming and swarming motility were regulated through c-di-GMP in S. Typhimurium and E. coli. (Boehm et al. 2010; Fang and Gomelsky 2010) Since the bcsE gene contains the c-di-GMP binding domain, we concluded that the effect of the gene in 


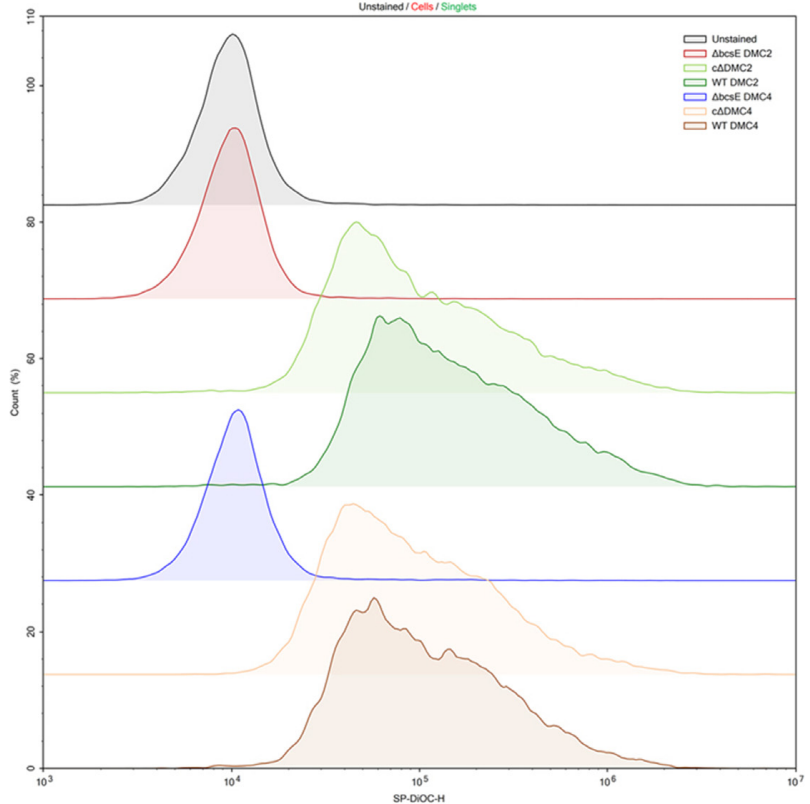

(A)

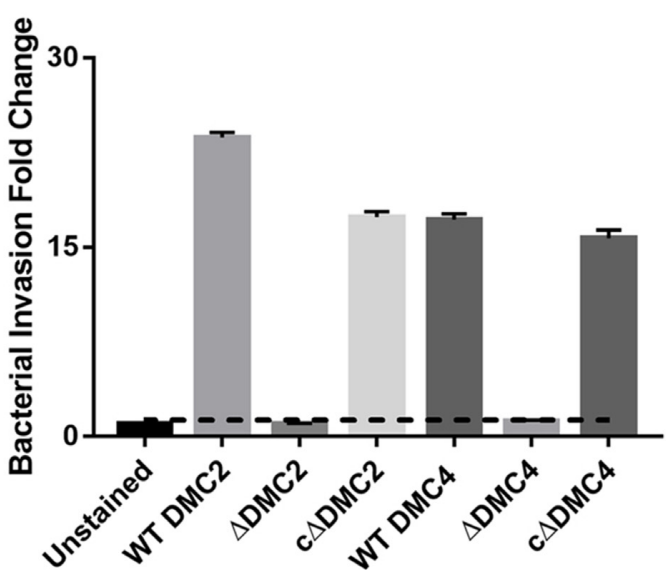

(B)

Figure 9. Quantities of intracellular DMC2, DMC4 and $\triangle b c s E$ DMC2, $\triangle b c s E$ DMC4 and bcsE gene complemented $c \triangle D M C 2$ and $c \triangle D M C 4$ strains determined using flow cytometry. (A) The ability of Salmonella invasion was determined by using the HT-29 cell line. Data show mean combined results of two independent experiments with three replicates. The invasion rate of bcsE mutant differed from that wild-type and bcsE gene complemented at $P<0.001$. (B) Columns represent to fold-change of strains of Salmonella invasion of the HT-29 cell line. Invasion of bcsE mutant stains all significantly decreased by $15-$ to 23 -fold compared to complementary and wild-type strains $(P<0.001)$.

question on S. Typhimurium motility is carried out in coordination with c-di-GMP. Our study is in line with the findings of the researchers. Because, in our study, it was observed that the swimming motility increased significantly in the mutants, where cellulose production decreased substantially. However, the mechanism of the swarming motility essentially protects its secret in bacteria. Our findings have a contribution to the literature in this sense. Because the high increase of swarming motility in bcsE gene mutants, where cellulose biosynthesis is significantly reduced, proves that BcsE protein negatively regulates the swarming motility. These findings shed light on the illumination of the mechanism of the bacterial swarming motility.

In our study, BcsE protein, it has been proven that the BscE protein induces an immune response in mice by strongly stimulating IgG1 and IgG2a humoral responses, as well as Th1 cellular responses. The ratios of IgG2a/IgG1 higher than 1 in mice immunized with pure BcsE protein showed that T lymphocyte type 1 (Th1) cytokine response was strong. The bcsE gene contributes significantly to Salmonella pathogenicity. Because the production of both humoral and strong cellular responses against BcsE protein is considered a general host response to pathogen invasion (Hall and Lee 2018). In studies conducted in mouse model systems, it was determined that Th1 responses had a specific role in protecting against Schistosoma mansoni infection. The same situation was determined for different pathogen-related molecular elements (PAMPs) for Salmonella infections. In these studies, it was determined that the dominant immune response type of mammalian host systems in the bacterial invasion stage was activation of Th1 cytokine production (Firacative et al. 2018). When all these literature data are evaluated together with the immune response findings obtained from our experiments, it turns out that BcsE protein plays an essential role in Salmonella infection. These findings are the first data on the subject in the literature.

In the second step, after determining that the BscE protein stimulates the immune response in mice, it was investigated whether the gene encoding the protein in question affects the persistence of the pathogen in mice. For this purpose, BALB/C mice were used as the model system. The number of viable cells in the spleen samples collected on day 1 and day 3 after infection with natural strains and their bcsE gene mutants remained as high as $10^{3}-10^{7} \mathrm{CFU} / \mathrm{g}$, while no live bacteria were detected in the counts for bcsE mutants. The number of mutant strains was 50 100 times lower for the same periods in liver samples. These data prove that the $b c s E$ gene is a very significant factor in the persistence of Salmonella in mammalian systems. The high persistence of the pathogen in the host system is defined as one of the most critical factors affecting virulence. The BcsE protein brought this persistence ability to Salmonella strains were not examined in our study. However, it is known that signal pathways play an essential role in adapting bacteria to changing environmental conditions (such as change of host and host system conditions). The systemic persistence effect occurs primarily when signal pathways activate the bacterial virulence factors and suppress host anti-virulence factors. The BscE protein contains the c-diGMP binding domain suggests that the effect is likely to regulate the c-di-GMP levels by the BscE protein. Because c-di-GMP levels result in activation or inactivation of the c-di-GMP signal pathway, which plays a crucial role in regulating cellulose biosynthesis, this plays a critical role in the systemic persistence of the pathogen as well as mobility (Le Guyon et al. 2015; Ahmad et al. 2016). However, this prediction needs to be verified. Testing the systemic persistence characteristics of the pathogen due to the change of c-di-GMP levels, especially in the presence and 
absence of the BcsE protein, will allow for precise prediction in question. Diagnosis of such systems that support acute infections is essential in preventing infections (Römling, Galperin and Gomelsky 2013). Therefore, the determination that the bcsE gene has an essential role in the systemic persistence of Salmonella, although its mechanism of action has not been elucidated, suggests that it may be possible to reduce in practice Salmonella virulence by using BcsE protein inhibitors.

In the last stage of this study, it was investigated whether the bcsE gene affects the epithelial cell invasion ability of the pathogen. For this purpose, HT-29 cells, a human intestinal epithelium cell line, were used. While the invasion rates of wildtype S. Typhimurium (DMC4) and S. Group C1 (DMC2) strains to HT-29 cells were obtained at $26.2 \%$ and $24 \%$, respectively, bcsE gene deleted mutants of these strains did not internalize HT29 cells. These findings are evidence that the bcsE gene also plays a role in host cell invasion. Invasion of epithelial cells of Salmonella constitutes the first stage of the pathogenicity process (Galán 1996). Cellulose is known to reduce host epithelial cell invasion, especially in Salmonella strains with high c-di-GMP levels described in studies conducted with E. coli (Wang et al. 2006). Later, Lamprokostopoulou et al. (2010) studied the deletion of the bcsA gene encoding the cellulose synthase enzyme and determined to have high c-di-GMP levels; epithelial cell invasion was reduced at least twice as compared to wild-type strains. These literature data shed light on explaining the findings we obtained from the invasion studies we conducted over the bcsE gene. In our study, it was determined that the bcsE gene significantly reduced cellulose biosynthesis. On the other hand, it is known that the BcsE protein is a c-di-GMP binding protein. Therefore, since the BcsE protein cannot be produced in the bcsE gene inactivation, c-di-GMP accumulates in mutants much higher than the wild-type strains. As the regulation of the level of c-di-GMP will not occur and mainly block cellulose synthesis. This negatively affects epithelial cell invasion of the pathogen, as shown in the literature data summarized above. However, the main difference of our study with the literature data in question is that the invasion ability in the bcsE gene mutants has completely disappeared but restored after bcsE complementation. This result indicates that the $b c s E$ gene is more effective than other genes known in an invasion.

As a result, the bcsE gene has proven to play active roles in Salmonella such as biofilm production, biofilm morphotype, mobility, cellulose and cellulase enzyme production, stimulation of host cell immune responses, epithelial cell invasion and persistence (acute infection formation) in host systems.

Conflict of interest. The authors declare no conflict of interest with this research.

\section{REFERENCES}

Ahmad I, Rouf SF, Sun L et al. BcsZ inhibits biofilm phenotypes and promotes virulence by blocking cellulose production in Salmonella enterica serovar Typhimurium. Microb Cell Fact 2016;15:177.

Allerberger F, Liesegang A, Grif K et al. Occurrence of Salmonella enterica serovar Dublin in Austria. Wiener Medizinische Wochenschrift 2003;153:148-52.

Barlow M, Hall BG. Origin and evolution of the AmpC $\beta$ Lactamases of Citrobacter freundii. Antimicrob Agents Chemother 2002;46:1190-8.
Beuzón CR, Holden DW. Use of mixed infections with Salmonella strains to study virulence genes and their interactions in vivo. Microbes Infect 2001;3:1345-52.

Boehm A, Kaiser M, Li $\mathrm{H}$ et al. Second messengermediated adjustment of bacterial swimming velocity. Cell 2010;141:107-16.

Cuatrecasas P. Protein purification by affinity precipitation. J Biol Chem 1970;245:3059-65.

Datsenko KA, Wanner BL. One-step inactivation of chromosomal genes in Escherichia coli K-12 using PCR products. Proc Natl Acad Sci 2000;97:6640-5.

Fang F, Lu WT, Shan Q et al. Characteristics of extracellular polymeric substances of phototrophic biofilms at different aquatic habitats. Carbohydr Polym 2014a;106:1-6.

Fang X, Ahmad I, Blanka A et al. GIL, a new c-di-GMP-binding protein domain involved in regulation of cellulose synthesis in enterobacteria. Mol Microbiol 2014b;93:439-52.

Fang X, Gomelsky M. A post-translational, c-di-GMP dependent mechanism regulating flagellar motility. Mol Microbiol 2010;76:1295-305.

Firacative C, Gressler AE, Schubert $\mathrm{K}$ et al. Identification of $\mathrm{T}$ helper (Th)1- and Th2-associated antigens of Cryptococcus neoformans in a murine model of pulmonary infection. Sci Rep 2018;8:1-14.

Flemming H-C, Wingender J. The biofilm matrix. Nat Rev Microbiol 2010;8:623-33.

Galán JE. Molecular genetic bases of Salmonella entry into host cells. Mol Microbiol 1996;20:263-71.

Gillespie IA, O'Brien SJ, Adak GK et al. Foodborne general outbreaks of Salmonella Enteritidis phage type 4 infection, England and Wales, 1992-2002: where are the risks? Epidemiol Infect 2005;133:795-801.

Hall CL, Lee VT. Cyclic-di-GMP regulation of virulence in bacterial pathogens. Wiley Interdiscip Reu RNA 2018;9:1-19.

Laemmli UK. Cleavage of structural proteins during the assembly of the head of bacteriophage T4. Nature 1970;227:680-5.

Lamprokostopoulou A, Monteiro C, Rhen M et al. Cyclic di-GMP signalling controls virulence properties of Salmonella enterica serovar Typhimurium at the mucosal lining. Environ Microbiol 2010;12:40-53.

Lamps LW. Infective disorders of the gastrointestinal tract. Histopathology 2007;50:55-63.

Le Guyon S, Simm R, Rehn M et al. Dissecting the cyclic di-guanylate monophosphate signalling network regulating motility in Salmonella enterica serovar Typhimurium. Environ Microbiol 2015;17:1310-20.

Mah TFC, O'Toole GA. Mechanisms of biofilm resistance to antimicrobial agents. Trends Microbiol 2001;9:34-9.

Majowicz SE, Musto J, Scallan E et al. The global burden of nontyphoidal Salmonella gastroenteritis. Clin Infect Dis 2010;50: 882-9.

Pui CF, Wong WC, Chai LC et al. Multiplex PCR for the concurrent detection and differentiation of Salmonella spp., Salmonella Typhi and Salmonella Typhimurium. Trop Med Health 2011;39:9-15.

Römling U, Galperin MY, Gomelsky M. Cyclic di-GMP: the first 25 years of a universal bacterial second messenger. Microbiol Mol Biol Rev 2013;77:1-52.

Römling U, Rohde M, Olsén A et al. AgfD the checkpoint of multicellular and aggregative behaviour in Salmonella typhimurium regulates at least two independent pathways. Mol Microbiol 2000;36:10-23. 
Römling U, Rohde M. Flagella modulate the multicellular behavior of Salmonella Typhimurium on the community level. FEMS Microbiol Lett 1999;180:91-102.

Römling U. Characterization of the rdar morphotype, a multicellular behaviour in Enterobacteriaceae. Cell Mol Life Sci 2005;62:1234-46.

Serra DO, Richter AM, Hengge R. Cellulose as an architectural element in spatially structured Escherichia coli biofilms. J Bacteriol 2013;195:5540-54.

Silva J, Leite D, Fernandes $M$ et al. Campylobacter spp. as a foodborne pathogen: a review. Front Microbiol 2011;2:1-12.

Solano C, García B, Valle J et al. Genetic analysis of Salmonella Enteritidis biofilm formation: critical role of cellulose. Mol Microbiol 2002;43:793-808.

Steele-Mortimer O. Infection of epithelial cells with Salmonella enterica. Methods Mol Biol 2007;431:201-11.

Steenackers H, Hermans K, Vanderleyden J et al. Salmonella biofilms: an overview on occurrence, structure, regulation and eradication. Food Res Int 2012;45:502-31.
Stepanović S, Vuković D, Dakić I et al. A modified microtiter-plate test for quantification of staphylococcal biofilm formation. J Microbiol Methods 2000;40:175-9.

Wang X, Rochon M, Lamprokostopoulou A et al. Impact of biofilm matrix components on interaction of commensal Escherichia coli with the gastrointestinal cell line HT-29. Cell Mol Life Sci 2006;63:2352-63.

Xie H, Gursel I, Ivins BE et al. CpG oligodeoxynucleotides adsorbed onto polylactide-co-glycolide microparticles improve the immunogenicity and protective activity of the licensed anthrax vaccine. Infect Immun 2005;73: 828-33.

Zogaj X, Nimtz M, Rohde M et al. The multicellular morphotypes of Salmonella Typhimurium and Escherichia coli produce cellulose as the second component of the extracellular matrix. Mol Microbiol 2001;39:1452-63.

Zorraquino V, García B, Latasa C et al. Coordinated cyclic-Di-GMP repression of Salmonella motility through ycgR and cellulose. J Bacteriol 2013;195:417-28. 